Revue trimestrielle sur l'image géographique et les formes du territoire

\title{
Entre Rhin et Jura, des espaces frontaliers où émergent des dissymétries spatiales
}

Alexandre Moine et Bernard Reitel

\section{OpenEdition}

Journals

Édition électronique

URL : http://journals.openedition.org/mappemonde/3966

DOI : 10.4000/mappemonde.3966

ISSN : 1769-7298

Éditeur

UMR ESPACE

Référence électronique

Alexandre Moine et Bernard Reitel, «Entre Rhin et Jura, des espaces frontaliers où émergent des dissymétries spatiales », Mappemonde [En ligne], 128 | 2020, mis en ligne le 01 juillet 2020, consulté le 15 septembre 2020. URL : http://journals.openedition.org/mappemonde/3966

Ce document a été généré automatiquement le 15 septembre 2020

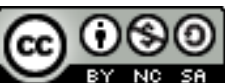

La revue Mappemonde est mise à disposition selon les termes de la Licence Creative Commons Attribution - Pas d'Utilisation Commerciale - Partage dans les Mêmes Conditions 4.0 International. 


\title{
Entre Rhin et Jura, des espaces frontaliers où émergent des dissymétries spatiales
}

\author{
Alexandre Moine et Bernard Reitel
}

\section{Des territoires nationaux dans une perspective d'intégration européenne}

1 L'article «Entre Rhin et Jura, des espaces frontaliers où émergent des dissymétries spatiales » a été écrit dans un contexte de dévaluation des frontières qui est une des composantes de la construction européenne (Foucher, 2000). L'instauration de l'UE en 1993 et la création de l'espace Schengen ont conduit à l'émergence d'un double processus d'intégration, l'un qualifié de "négatif», à travers la disparition des contrôles sur les frontières "internes" et l'autre qualifié de "positif», grâce à l'élaboration d'un cadre de normes communes à l'échelle supranationale (Scharpf, 1999) dont la politique de cohésion offrirait un exemple notable. La signature des accords bilatéraux en 1999 et en 2004 et la participation de la Suisse à certaines politiques de l'UE traduisent cependant une intégration significative, mais sélective entre ces deux entités.

2 Quinze ans plus tard, ce cadre général est réinterrogé, voire remis en cause alors que dans le même temps, l'intégration fonctionnelle n'a jamais été aussi intense (Kahn, 2017). Des flux de différentes natures traversent les frontières, dont certains, qui relient des lieux géographiquement proches situés dans des pays limitrophes, sont à proprement parler transfrontaliers. Dans ce cadre, deux dynamiques contradictoires sont observables dans les régions frontalières. D'une part, les programmes de coopération transfrontalière Interreg instaurés en 1990 et qui en sont à leur cinquième phase (2014-2020) ont permis de tisser des liens de part et d'autre des frontières nationales (Wassenberg et al., 2015). D'autre part, la coopération s'est essoufflée ou a 
fait l'objet d'un recentrage sur certaines orientations dans plusieurs régions d'Europe de l'Ouest (Beck, 2019).

Les systèmes politiques et institutionnels des trois pays considérés, Allemagne, France et Suisse ont connu des évolutions sans qu'on puisse cependant parler de convergence : les cultures nationales demeurent fondamentalement distinctes (Vaillant, 2015). La systématisation de la planification aux échelles régionale et infrarégionale en France n'a pas conduit à une diminution des différences avec les systèmes des États voisins en dépit de l'émergence de préoccupations similaires (enjeux démographiques, environnementaux, énergétiques), de la diffusion de pratiques analogues de gouvernance (Allmendinger et al., 2015 ; Desjardins et Béhar, 2017), et compte tenu de l'absence de base légale intégrant la dimension transfrontalière dans les documents de planification en France et en Suisse.

Carte. Évolution des aménagements routiers et ferroviaires entre 2005 et 2019

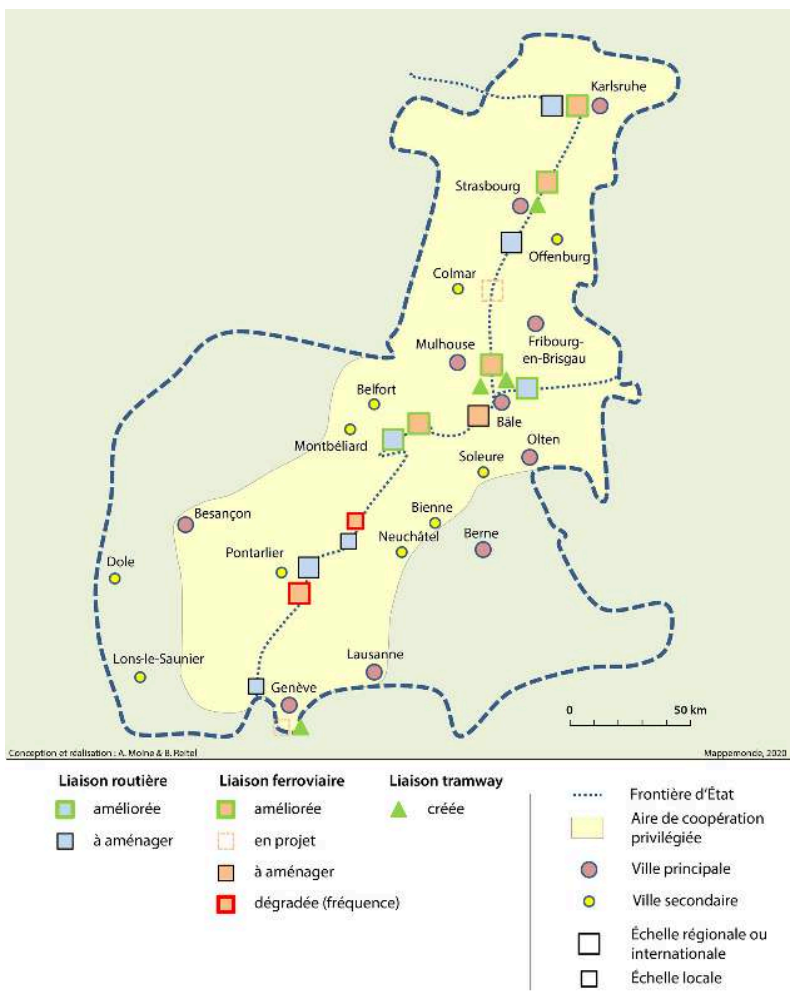

\section{Une intégration fonctionnelle, des systèmes discontinus de planification}

Dans chacun des cas d'études, les composantes françaises ont été intégrées en 2014 dans des régions reconfigurées (3 lois votées en 2014 et 2015, délimitation des régions, MAPTAM, NOTRe), l'Alsace dans le Grand Est et la Franche-Comté dans la BourgogneFranche-Comté. Dans un contexte où l'intégration fonctionnelle transfrontalière est marquée, ces fusions n'ont, pour l'instant, pas eu d'influence majeure sur la prise en compte des dimensions transfrontalières.

Les accords bilatéraux sur la libre circulation des personnes (ALCP de 2002) ont permis d'atténuer les effets de la non-adhésion de la Suisse à l'UE. Leur remise en question par 
un référendum en 2014 concerne plus de 145000 travailleurs en Suisse, 32000 frontaliers de l'Arc jurassien et 69000 dans le Rhin supérieur qui compte aussi 25000 actifs résidant en France et qui sont employés en Allemagne. Ces flux sont représentatifs des nombreuses interactions qui existent de part et d'autre de la frontière dans les deux régions transfrontalières (frontaliers, tourisme d'achat, marchandises) et qui construisent des continuités fonctionnelles qui tendent à effacer la frontière (Moine, 2012).

6 Par ailleurs, les outils d'aménagement des trois territoires, allemands, français et suisse demeurent très différenciés tant d'un point de vue des procédures juridiques que de la manière de les mettre en œuvre. Mais, cette situation génère de multiples problématiques territoriales: étalement urbain côté français, saturation des axes de transport routier, difficultés pour les commerces et les services côté Suisse, etc. Par ailleurs, il s'avère que les normes politico-administratives ne sont absolument pas harmonisées entre la France et la Suisse, les droits nationaux prévoyant l'indépendance des législations en matière de droit de propriété. L'intégration est plus avancée dans le Rhin supérieur, entre France et Allemagne : les documents de planification intègrent les visions des territoires frontaliers limitrophes, mais les temporalités différentes empêchent une co-construction d'une vision commune. Seule l'agglomération bâloise a pour l'instant réussi à imaginer un plan général d'aménagement dans le cadre de l'Eurodistrict Trinational de Bâle (ETB), mais sa démarche relève plus du soft planning que d'une planification territoriale.

\section{Une intégration différenciée des réseaux transfrontaliers}

7 L'intégration transfrontalière des réseaux de circulation a connu des évolutions assez distinctes dans les deux régions transfrontalières : les discontinuités se sont en grande partie atténuées dans le Rhin supérieur, alors que dans l'Arc Jurassien elles demeurent relativement marquées.

8 L'amélioration est particulièrement notable dans le domaine ferroviaire dans le Rhin supérieur, ce qui a permis de résorber certains points noirs et désengorger certains axes. L'instauration du TGV Est en 2007 et la mise en service de liaisons à grande vitesse ont conduit à une réduction significative des temps de parcours entre les principales villes alsaciennes et Paris ainsi qu'avec les grandes métropoles du Nord et de l'ouest du pays et Bruxelles. De nouvelles améliorations ont été apportées en 2011 avec la mise en service du TGV Rhin-Rhône qui assure de meilleures relations entre le sud-ouest de l'Allemagne, le nord-ouest de la Suisse et les villes d'Alsace et de l'Arc Jurassien ainsi qu'avec Lyon et Marseille. Par ailleurs, une meilleure continuité a été assurée sur le plan ferroviaire à l'échelle régionale au sein du Rhin supérieur grâce à l'augmentation des fréquences. La SNCF a adopté dès 2006 un système de cadencement des trains express régionaux (TER) en Alsace afin de se coordonner avec les systèmes ferroviaires allemand et suisse limitrophes. L'amélioration conforte les principaux pôles urbains, Strasbourg, Bâle, Mulhouse, Karlsruhe et Fribourg. La création du Grand Est interroge cependant la pérennité des améliorations sur le réseau régional puisque la région doit penser l'avenir d'un réseau ferré régional à l'échelle de son nouveau territoire sans être dotée de moyens supplémentaires. Enfin, certaines améliorations significatives ont été apportées au sein des agglomérations transfrontalières, ce qui permet de renforcer leur 
caractère métropolitain (Reitel, Perrin, Pupier, 2017) : deux lignes de tramway ont été prolongées dans l'ETB, l'une vers l'Allemagne (Weil am Rhein en 2015) et l'autre vers la France (Saint-Louis en 2017) et une ligne de tramway a été prolongée de Strasbourg à Kehl en 2 étapes en 2017 et en 2019. Les inaugurations de ces extensions ont eu un fort impact symbolique, car elles matérialisent non seulement une continuité qui remet en cause la frontière en tant que limite de séparation, mais aussi, car leurs constructions se sont faites au moment où des contrôles étaient réintroduits de manière régulière aux postes-frontière sur les voies routières au sein des agglomérations. En revanche, le réseau routier continue de présenter des discontinuités marquantes, notamment dans le franchissement de la frontière entre Alsace et Palatinat. La dimension symbolique de l'intégration est d'autant plus forte lorsqu'on considère qu'entre 2004 et 2019, pas moins de 4 ponts ont été construits sur le Rhin (dont deux passerelles piétons-cyclistes) qui est le support du tracé de la frontière entre France et Allemagne et sur une grande partie entre Allemagne et Suisse.

Dans l'Arc jurassien, les discontinuités relevées en 2005 ont évolué, mais dans une sorte de jeu à somme nulle d'un point de vue des transports ferroviaires, puisqu'à la réouverture d'une ligne (Delle - Belfort suspendue en 1997 puis rouverte en décembre 2018) a correspondu la disparition de cadencement sur d'autres (suppression du Lyria vers Neuchâtel et plus récemment suppression d'un train sur la même ligne vers Lausanne depuis Paris). Par ailleurs la réouverture de la ligne Delle - Meroux-Moval Belfort fait l'objet de critiques quant aux horaires proposés qui n'ont pas permis un report modal efficace des frontaliers qui continuent de privilégier la mobilité routière et par rapport à l'insuffisance du cadencement jusqu'à Belfort. Globalement, la dissymétrie entre les réseaux routiers français et suisses demeure, voire s'est accentuée avec la livraison de l'autoroute Transjurane (A16) entre Bienne (CH) et Boncourt (CH)/ Delle (F) en 2017. La prochaine mise en travaux du contournement du Locle et de La Chaux-de-Fonds par la H2O ouvrira un nouvel accès rapide vers la France dans le Canton de Neuchâtel via le Col des Roches, mais sans amélioration prévue en vis-à-vis français. Sur le même axe, en transport ferré, la ligne transfrontalière des « Horlogers » fait l'objet d'améliorations programmées entre Besançon et la frontière au Col des Roches au travers d'une assez forte mobilisation de la Région sur ce dossier.

\section{Une difficile intégration institutionnelle}

10 Sur le plan politique, un grand nombre de structures de coopération transfrontalière existe aux échelles locales et infrarégionales, mais certaines d'entre elles s'interrogent sur leur avenir. La création d'un nouvel instrument juridique tel que le Groupement Européen de Coopération Transfrontalière (GECT) qui révèle a priori l'existence d'une intégration institutionnelle ne traduit pas forcément une réelle intégration politique et peut même être la source de difficultés inédites. La coopération est à la fois source de rapprochement et de tensions qui résultent soit des différences administratives ou politiques, soit des difficultés de se comprendre (interculturalité et langues).

11 Dans le Rhin supérieur, deux échelles ont fait l'objet d'un investissement soutenu par les pouvoirs publics. Une stratégie métropolitaine trinationale constituée de 4 piliers sectoriels (économie, sciences, société civile, politique) a été élaborée à partir de 2006 en vue de coordonner les actions à cette échelle. La démarche validée par un accord 
intergouvernemental en 2010 permet de développer des projets grâce à l'existence de fonds propres et de financements par les programmes Interreg.

Les agglomérations urbaines transfrontalières constituent indéniablement des zones d'intégration transfrontalière (organisation de manifestations et de projets communs, gestion partagée, etc.). Pourtant Strasbourg et Bâle ont adopté des formes de gouvernance différentes. La première a créé un GECT en 2010 qui correspond à l'Eurodistrict qui avait été mis en œuvre sous l'impulsion des deux États français et allemand en 2003. Les principales opérations sont cependant plus l'œuvre d'une coopération bilatérale entre l'Eurométropole de Strasbourg et la ville voisine de Kehl que de l'Eurodistrict. La seconde a conservé une forme institutionnelle souple, celle d'une association (Walther \& Reitel, 2013), ce qui n'a pas empêché, bien au contraire, le développement de plusieurs projets d'envergure: organisation d'une grande manifestation d'architecture et d'urbanisme en 2020 (Internationale BauAustellung Basel 2020 - IBA -), réaménagement des espaces portuaires à la confluence des Troisfrontières (projet Dreiland), etc.). Les difficultés d'articulation restent toutefois réelles du fait de l'existence de trois systèmes nationaux.

La gouvernance transfrontalière dans l'Arc jurassien franco-suisse repose sur l'existence de la Conférence Transjurassienne créée en 1985, qui a proposé en 2016 une nouvelle stratégie de développement (Conférence Transjurassienne, 2016). Pour la partie française, l'État est toujours présent dans le dispositif aux côtés de la région Bourgogne - Franche-Comté en vis-à-vis des cantons de Jura, Berne, Neuchâtel et Vaud. On soulignera la montée en puissance d'une thématique jusqu'alors passée sous silence, le bien vivre ensemble qui s'accompagne d'une attention plus forte portée aux petites coopérations comme en témoigne la mise en place d'un (modeste) fonds consacré aux petits projets. Une récente étude du Forum Transfrontalier Arc jurassien pointait à ce titre l'importance des coopérations de petite taille (Moine, 2017) qui appellent, à l'échelle européenne, la création d'un fond spécifique en parallèle des crédits Interreg que l'on connaît. Enfin, un Groupement Local de Coopération Transfrontalier (GLCT) a vu le jour dans l'Arc jurassien, fondé sur la continuité urbaine entre le Val de Morteau et les villes du Locle et de La Chaux-de-Fonds pour un total d'environ 70000 habitants. Les envies politiques de collaborer sont présentes, mais malheureusement en l'absence de moyens financiers, le dispositif demeure une coquille vide.

\section{Des perspectives d'intégration à reconsidérer ?}

En définitive, les 3 interrogations évoquées en conclusion dans
toujours d'actualité, même si des nuances peuvent être apportées.

D'une part, la convergence entre les maillages territoriaux n'est pas vraiment à l'ordre du jour. Les fusions des régions et la réorganisation des structures intercommunales en France n'ont paradoxalement pas rapproché les collectivités régionales et locales de leurs homologues suisses ou allemands. Chaque État semble évoluer selon une logique qui lui est propre et cela ne semble pas près de changer. Cela étant, est-ce vraiment un obstacle à la coopération transfrontalière? Il semblerait qu'une bonne interconnaissance des systèmes territoriaux voisins et l'existence d'une volonté commune constituent un préalable à une bonne coopération transfrontalière. toujours sur l'existence d'une dimension juridique. L'intégration institutionnelle n'est 
pas une condition sine qua non d'une bonne intégration fonctionnelle. En revanche, une gouvernance qui assure un dialogue régulier et qui permet de résoudre les problèmes est nécessaire: elle nécessite à la fois une bonne intégration politique horizontale (entre pouvoirs publics de part et d'autre de la frontière) et une bonne articulation verticale (entre les États centraux et les collectivités des niveaux locaux et régionaux). En effet, certaines questions d'ordre juridique notamment ne peuvent être résolues que par les États centraux, y compris dans les États fédéraux et peuvent conduire à des redimensionnements de projets, à titre d'exemple, le pilotage institutionnel francosuisse du Parc Naturel Régional du Doubs et de son équivalent suisse a été refusé par l'État français. Un nouvel instrument juridique est toutefois envisagé à l'échelle européenne: l'European Cross-Border Cooperation (ECBC) prévoit d'autoriser l'application d'une norme nationale dans un territoire limitrophe en cas d'un projet transfrontalier (Mission Opérationnelle Transfrontalière, 2017).

Enfin, la logique des programmes Interreg s'inscrit dans des cadres spatiaux clairement délimités et qui n'ont pas évolué dans notre zone d'études, ce qui permet aux acteurs de se connaître et de tisser des liens, mais sans garantie de pérennité. Au-delà de la période de crédit, la mobilisation peut effectivement cesser. En revanche, des dialogues existent dans le cadre des périmètres des programmes de coopération transnationale (dans Interreg V, la CTJ et le Rhin supérieur se trouvent dans le programme Espace Alpin) et dans les stratégies macro-régionales (Région Alpes pour nos deux zones). L'enjeu consiste dès lors à poursuivre la programmation Interreg, dont une sixième phase est à présent actée, à considérer avec attention la mise en place d'un fonds dédié aux "petites coopérations transfrontalières", mais aussi et surtout à proposer des visions intégrées qui associent les acteurs publics à l'intérieur des États impliqués en les articulant à leurs interlocuteurs transfrontaliers autour des centralités périphériques que constituent les "territoires transfrontaliers" (Moine, 2019). Ceci nécessite certes des instruments et des dispositifs, mais aussi, et surtout un cadre idéologique propice à l'ouverture et au dialogue.

\section{BIBLIOGRAPHIE}

ALLMENDINGER P., HAUGHTON G., KNIELING J., OTHENGRAFEN F. (2015). Soft spaces in Europe. Re-negotiating governance, boundaries and borders. Londres : Routledge. BECK J. (2019). Transdisciplinary Discourses on Cross-Border Cooperation in Europe. Bruxelles : Peter Lang.

CONFÉRENCE TRANSJURASSIENNE (2016). Pour une nouvelle dynamique de l'Arc jurassien, Stratégie de coopération transfrontalière 2016-2020. Besançon : Augé.

DESJARDINS X., BÉHAR D. (2017). « Les régions françaises enfin aménageuses du territoire ? ». Population \& Avenir, n ${ }^{\circ}$ 733(2017-3), p. 17-19.

FOUCHER M. (2000). La République européenne. Paris : Belin. 
KAHN S. (2017). « Les enjeux de la crise européenne ». Hérodote, nº 164 (2017-1), p. 79-100.

MISSION OPÉRATIONNELLE TRANSFRONTALIÈRE. (2017). Les territoires transfrontaliers. La fabrique de l'Europe. Paris : Mission Opérationnelle Transfrontalière. En ligne : http://www.espacestransfrontaliers.org/fileadmin/user_upload/documents/Documents_MOT/Europe/ Positions_MOT/Position_post2020_FR_brochure_territoires_tf_MOT.pdf

MOINE A. (2012). « Les effets frontière dans l'Arc jurassien », Actes du colloque " L'Arc jurassien :

frontière ou interface ? ». Besançon : Fédération des Sociétés Savantes de Franche-Comté, Société d'Émulation du Doubs, Estimprim, p. 365-387.

MOINE A. (2017), « Les coopérations de proximité dans l'Arc jurassien franco-suisse : Un enjeu de la cohésion sociale transfrontalière ». In Revue géographique de l'Est, vol. 57, no 1-2. En ligne : http://journals.openedition.org/rge/6045

MOINE A. (2019). « Une frontière internationale comme catalyseur de dynamiques ? Le cas ambigu de l'Arc jurassien franco-suisse ». In B. CASTETS FONTAINE, M. KACI, J. LOISEAU, A. MOINE, Deux frontières aux destins croisés ? Étude interdisciplinaire et comparative des délimitations territoriales entre la France et la Suisse, entre la Bourgogne et la Franche-Comté (XIV siècle-XXI ${ }^{e}$ siècle), Besançon : Presses universitaires de Franche-Comté, coll. « Les cahiers de la MSHE », p. 211-223.

REITEL B., PERRIN T., PUPIER P. (2017). « La métropolisation transfrontalière ». In MOULLÉ F. Frontières. Bordeaux : Presses universitaires de Bordeaux, p. 205-228.

SCHARPF F. (1999). Governing in Europe: effective and democratic? Oxford : Oxford University Press. VAILLANT J. (2015). «Introduction. De la difficulté des réformes territoriales en France et en Allemagne ». Allemagne d'aujourd'hui, nº 212(2015-2), p. 62-69.

WALTHER O., REITEL B. (2013). « Cross-border policy networks in the Basel region: the effect of national borders and brokerage roles ». Space \& Polity, vol. 17, $n^{\circ} 2$, p. 217-236.

WASSENBERG B., REITEL B., PEYRONY J. (2015). Territorial cooperation in Europe. A historical perspective. Luxembourg : Publications Office of the European Union. En ligne : https://ec.europa.eu/ futurium/en/system/files/ged/interreg_25years_en.pdf

INDEX

Thèmes : 30 ans de publication dans Mappemonde 